\title{
Vibrotactile Feedback in Steering Wheel Reduces Navigation Errors during GPS-Guided Car Driving
}

\author{
Enes Selman Ege, Furkan Cetin, Cagatay Basdogan \\ Koc University
}

\begin{abstract}
We show that vibrotactile feedback displayed through the steering wheel of a car can reduce the perceptual and cognitive load of the driver, leading to less distraction and fewer navigation errors. To demonstrate the concept, two vibration motors are mounted onto the steering wheel of a driving simulator and driving experiments are performed in virtual environments under two different sensory conditions (auditory alone and auditory and vibrotactile feedback together). The results of our experiments with 12 subjects show that, if passenger auditory noise and distraction exist in the environment, the navigation errors (making a wrong turn or taking a wrong exit) are reduced when vibrotactile feedback is displayed to the users in tandem with the GPS-based voice commands.
\end{abstract}

KEYwORDS: vibrotactile, haptics, car navigation systems, GPS, steering wheel, driving simulator.

INDEX TeRMS: H.5.2. [Information Interfaces and Presentation]: Haptic User Interfaces - Haptic I/O

\section{INTRODUCTION}

The GPS-based navigation systems are helpful in finding our destination, and becoming a standard feature in today's cars. These systems utilize visual cues and voice commands to guide the driver during navigation. However, excessive visual information on the navigator screen overwhelms the driver. Moreover, conversation of the passengers with each other and the driver, as well as the external noise coming from the road or the environment can easily distract the driver and make the voice commands ineffective. Also, the users of these systems report that the artificial "computer" voice can be annoying. We believe that haptic feedback can decrease the cognitive workload of the driver and reduce the navigation errors. In particular, our aim is to show that the vibrotactile feedback improves the navigation performance of the driver and makes the driving task less exhausting, especially when passenger auditory noise and distraction exist in the environment.

The use of haptic feedback in vehicular settings is a relatively new concept and a review of the literature is available in $[1,2]$. There are studies showing the benefits of vibrotactile feedback in arousing sleepy drivers [2], alerting drivers to approaching danger [3], presenting more detailed navigational information [4], and reducing driver workload when interacting with in-vehicle devices, but we are not aware of any earlier study that aims to integrate vibrotactile feedback into a GPS-based car navigation system to improve the navigation performance of a driver. In [5], vibration motors were attached to the steering wheel of a driving simulator and the recognition rate of the drivers for the

College of Engineering, Koc University, Istanbul, Turkey,

34450. E-mail: enesege, fcetin, cbasdogan@ku.edu.tr

IEEE World Haptics Conference 2011

21-24 June, Istanbul, Turkey

978-1-4577-0297-6/11/\$26.00 @2011 IEEE vibrotactile stimulus coded in spatio-temporal patterns was measured, but its application to a GPS-based navigation system has not been considered at all. Like any other in-vehicle devices, GPS-based car navigation systems can have a negative effect on safety if they increase the cognitive workload of the driver or distract her/his attention [6,7 and 8]. It is known that visual and auditory information channels of the today's drivers are already highly occupied. With the availability of GPS-based navigation systems as a standard feature in today's cars, the driver has to concentrate on not only the road, mirrors, and the visual displays in the front panel, but also the voice commands coming from the navigator. Rather than overloading the visual and auditory channels, we investigate an alternative approach in which some of the navigation information is displayed through the haptic channel in order to alleviate the perceptual and cognitive load of the driver. The motivation behind this approach is obvious: our hands are sensitive to amplitude, duration, and frequency of a vibratory stimulus (see the guidelines in [9]). Furthermore, while the auditory and visual signals displayed by the navigation system may disturb the other passengers in the car, the tactile channel is private [10] and provides a direct connection with the driver. In this regard, the driver can respond to the haptic stimuli faster than the visual and auditory ones. This feature also helps when there is passenger noise and distraction in the car. If the passengers in the car make conversation with the driver and each other, the voice commands displayed from the navigator may easily distract the driver or may not be heard at all.

This paper a) introduces the operating principles of a vibrating steering wheel designed to reduce the navigation errors during GPS-guided car driving and b) presents the results of our human experiments performed with this steering wheel and a driving simulator. The goal of the experiments is to compare the navigation performances of the users under two sensory conditions: a) only auditory navigation cues (i.e. standard voice commands) are displayed to the user versus b) both auditory and haptic navigation cues are displayed together to the same user. Under both conditions, passenger auditory noise and distraction exist in the environment.

\section{METHOD}

\subsection{Set-up}

A driving simulator consisting of a simulation engine, steering wheel, and a pedal set (Trust, Model No: GM-3200) is developed (Figure 1). The simulation engine is written in Game Maker Pro Version 8.0. The steering wheel and the pedal set are connected to a PC via Universal Serial Bus (USB). Using the simulation engine, 2 different driving maps, consisting of two-way roads, intersections, roundabouts, and road junctions are constructed. The first map is used for training the subjects while the second one is used for the experiments. A potentiometer is attached to the shaft of the steering wheel to measure its rotational movements. Two vibration motors are mounted onto the steering wheel for 
displaying vibrotactile cues to the subjects as shown in Figure 1. There are mainly two types of vibratory motors: cylindrical (bar) and coin (button). In this project, coin type motors are preferred since their rotating eccentric load is enclosed in a case and they make the design more compact. The rated voltage and speed of the motors are $3 \mathrm{~V}$ and $12000 \mathrm{rpm}$, respectively (1034A, Kysan Electronics). To control the motors, an electronic circuit including a microcontroller (PIC 16F877A) is designed. The microcontroller has an output current of about $15 \mathrm{~mA}$, which is not sufficient to drive the motors. For this reason, two high-current-output transistor-arrays (ULN2803-D, $500 \mathrm{~mA}$ ) are used, which are driven by the microcontroller.
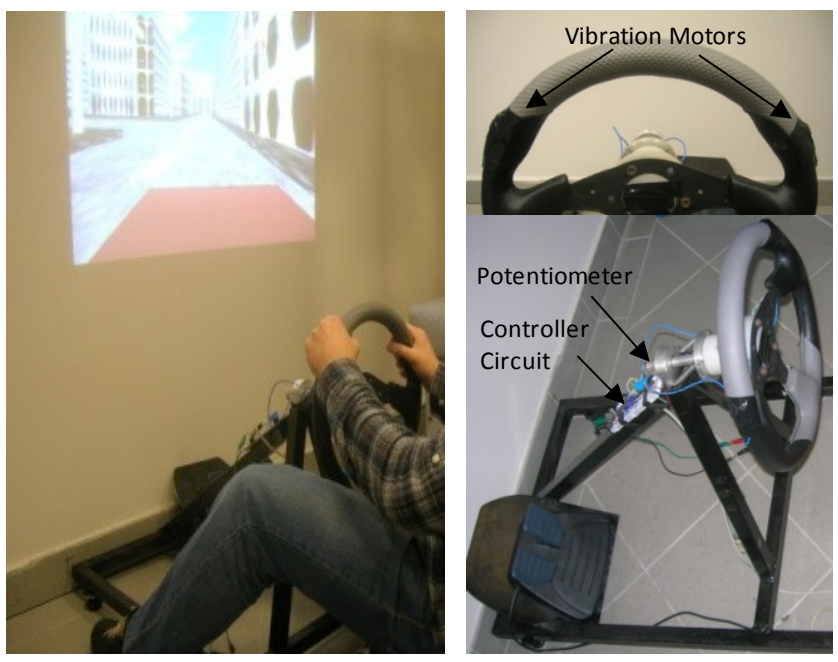

Figure 1. Our driving simulator: 2 vibration motors are mounted onto the steering wheel of the simulator to display vibrotactile stimulus to the user for navigational guidance.

\subsection{Auditory and Tactile Navigation Signals}

The auditory signals used in our system are the standard GPSbased voice commands. The corresponding vibrotactile commands are produced by the two vibration motors mentioned above. Table 1 tabulates the voice commands mapped to the vibrotactile signals (Table 1).

Table 1. Voice commands and their corresponding vibrotactile signals

\begin{tabular}{ll}
\hline Voice command & Corresponding vibrotactile signal \\
\hline Turn right & $\begin{array}{l}\text { The motor on the right vibrates continuously } \\
\text { until the turning operation is over. }\end{array}$ \\
\hline Turn left & $\begin{array}{l}\text { The motor on the left vibrates continuously } \\
\text { until the turning operation is over. }\end{array}$ \\
\hline $\begin{array}{l}\text { Approaching } \\
\text { a roundabout }\end{array}$ & $\begin{array}{l}\text { Both motors start vibrating continuously } \\
\text { until the exit. }\end{array}$ \\
\hline $\begin{array}{l}\text { Leave the } \\
\text { roundabout }\end{array}$ & Both motors stop vibrating. \\
\hline Go ahead & - \\
\hline
\end{tabular}

\subsection{Subjects}

A total of 12 subjects ( 2 female, 10 male) participated in the study. Their age is $24.1 \pm 1.4$ years. All subjects have a driver's license and normal vision. They also have experience with computer games (average $=3.2 \pm 1.1$, based on a scale of 1 : lowest to 5: highest)

\subsection{Experimental Procedure}

The subjects are asked to drive a virtual car from location A to location B on a given map while holding the steering wheel with both hands from the locations where the motors are mounted. Before the experiment, they are given an orientation about the purpose of the study, how to use the steering wheel and the pedals, and the meaning of the tactile signals. After the orientation and before the test trial, they are educated to differentiate the tactile signals coming from the left and right-hand sides of the steering wheel. For this purpose, a vibrotactile stimulus is displayed to the subjects a total of 10 times through the motor either on the left or the right side of the steering wheel in random order (5 times each) and they are asked to differentiate which side the signal is coming from. The subjects are allowed to proceed to the test trial only if their success rate in the tactile test is above $70 \%$. During the test trial, each subject is asked to navigate on the first map for 5 minutes in order to get familiar with the simulator. Then, the subjects are tested on the second map under the following sensory conditions:

(1) only auditory feedback (A)

(2) auditory and vibrotactile haptic feedback (AH)

In auditory-haptic feedback condition, the beginning of vibrotactile signals are synchronized with that of the auditory navigation signals. The vibrotactile signals are displayed until the driving maneuver is over. The subjects are divided into two groups randomly and a matrix design is followed to reduce the learning effects. Each subject in a group repeats the experiment 3 times. The results presented in this paper are the averages of those 3 trials. The first group received condition (1) first, and then two weeks later repeated the experiment under condition (2). The second group, received condition (2) first, and then participated in the auditory feedback session (condition 1) two weeks later (see Table 2).

Table 2. Grouping of the subjects in our experiment

\begin{tabular}{l|l|l}
\hline & Group 1 (6 subjects) & Group 2 (6 subjects) \\
\hline $\begin{array}{l}\text { First } \\
\text { Session }\end{array}$ & Auditory feedback & $\begin{array}{l}\text { Auditory-haptic } \\
\text { feedback }\end{array}$ \\
\hline \multicolumn{3}{|c}{ Two weeks break } \\
\hline $\begin{array}{l}\text { Second } \\
\text { Session }\end{array}$ & $\begin{array}{l}\text { Auditory-haptic } \\
\text { feedback }\end{array}$ & Auditory feedback \\
\hline
\end{tabular}

To test our hypothesis that the car drivers are less affected by the auditory noise and distraction when GPS-based navigation commands are augmented by vibrotactile cues, city noise (i.e. police and ambulance sirens, car horns, and engine noise) and background music are displayed to the subjects through two separate speakers during the experiment (Table 3 ). In addition, the experimenter, playing the role of a passenger sitting next to the driver in the car, distracts the driver by continuously asking her/him questions from a standard questionnaire and trying to initiate a conversation with the driver during the experiment. Subjects are instructed to answer the questions asked by the experimenter. Thus, a continuous auditory distraction due to the passenger noise is also simulated during the experiment.

Table 3. Auditory noise levels in our driving simulator

\begin{tabular}{ll}
\hline City noise & $\sim 60 \mathrm{~dB}$ (average) \\
\hline City noise + Voice Commands & $\sim 72 \mathrm{~dB}$ (peak) \\
\hline City noise + Background Music & $\sim 75 \mathrm{~dB}$ (average) \\
\hline $\begin{array}{l}\text { City noise + Background Music } \\
+ \text { Passenger Noise }\end{array}$ & $\sim 85 \mathrm{~dB}$ (average) \\
\hline
\end{tabular}




\section{RESULTS}

\subsection{Quantitative Measures}

To reach the destination in the second map, it was required to make 9 right turns, 8 left turns and to take 2 roundabout exits. For each trial, we recorded the number of times the subjects made a wrong-turn or took a wrong-exit on a roundabout. When the subjects made a wrong turn or took a wrong exit on a roundabout, it was counted as a navigation error and the subject was transported back to the location and time where she/he received the last navigation signal. The transportation was necessary to prevent the subjects from getting lost in the map and loosing additional time.

The vibrotactile feedback improved the navigation performance of all the subjects who participated in the experiment as shown in Fig. 2a. On the average, the number of navigation errors the subjects made was $3.39 \pm 1.24$ and $0.92 \pm 0.81$ under the auditory condition alone (A) and auditory and haptic conditions together $(\mathrm{AH})$, respectively (Fig. 2b). This result suggests that the subjects navigated better when vibrotactile cues were displayed to them in addition to the auditory ones $(\mathrm{p}<0.001)$. These results support the earlier finding that a localized vibration coming from the driver seat is an intuitive way to present direction information in navigation [4].

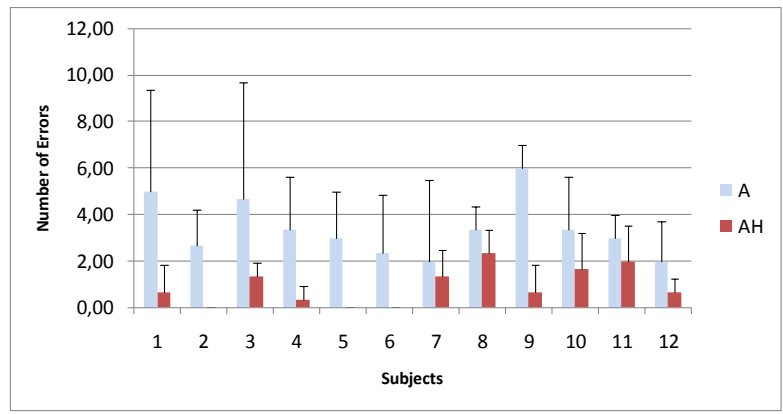

a)

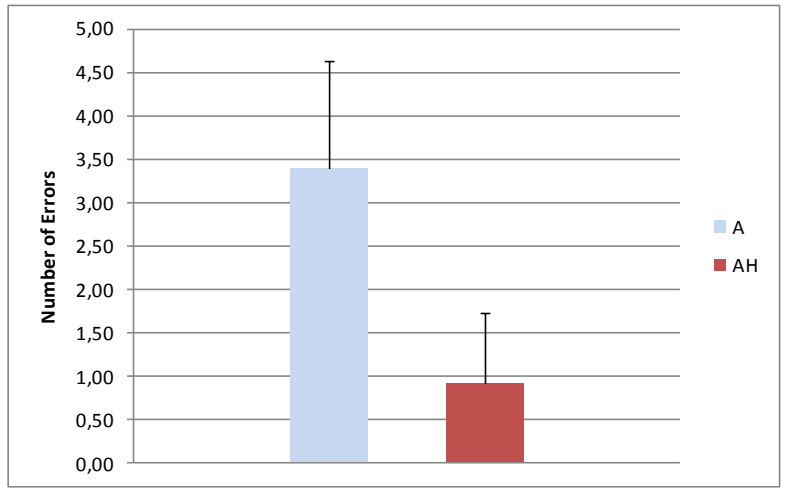

b)

Figure 2. a) The number of navigation errors for each subject under auditory and auditory-haptic feedback conditions, b) the average of all subjects. The bars represent the standard deviations.

We further analyzed the data to see if there was any difference between the experimental sessions and the groups (see Table 2). As shown in Figure 3, the subjects in each group made fewer errors in the second session than the first one since they became familiar with the experimental set-up and the driving path (note that the labels "first timers" and "second timers" on the x-axis represent the subjects who performed one of the two conditions for the first or second time, respectively). However, independent of the sequence of the sessions, the subjects made fewer errors in navigation when vibrotactile cues were displayed to them. Moreover, there was no significant difference in the navigation errors of the groups 1 and 2 under each sensory condition $(\mathrm{p}>$ 0.1 ), which enabled us to combine the data of both groups into a single data set for each condition to display the results shown in Fig 2b.

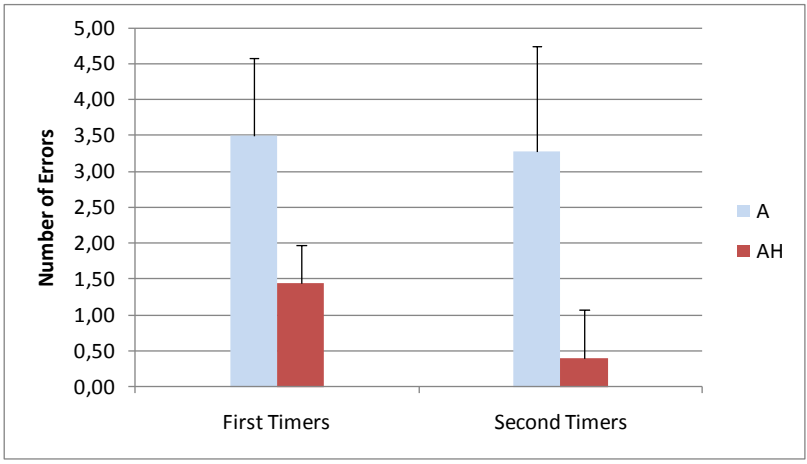

Figure 3. The average number of navigation errors under auditory and auditory-haptic feedback conditions for the first and second sessions. The bars represent the standard deviations.

We also investigated if there was any correlation between the number of navigation errors and the task completion time in our experiments. In particular, we investigated if the driving speed of the user had any influence on her/his navigation errors. The average task completion times of the subjects were $210.7 \pm 31.9$ and $206.0 \pm 25.3$ seconds under auditory and auditory-haptic conditions, respectively and there was no significant difference between them $(\mathrm{p}>0.1)$.

Moreover, as shown in Figure 4, the correlation between the number of navigation errors and the task completion time was also low, suggesting that the driving speed had no influence on the navigation errors in our experiments (the correlation coefficients are $r=0.42$ and $r=0.43$ for the auditory and auditory-haptic conditions, respectively)

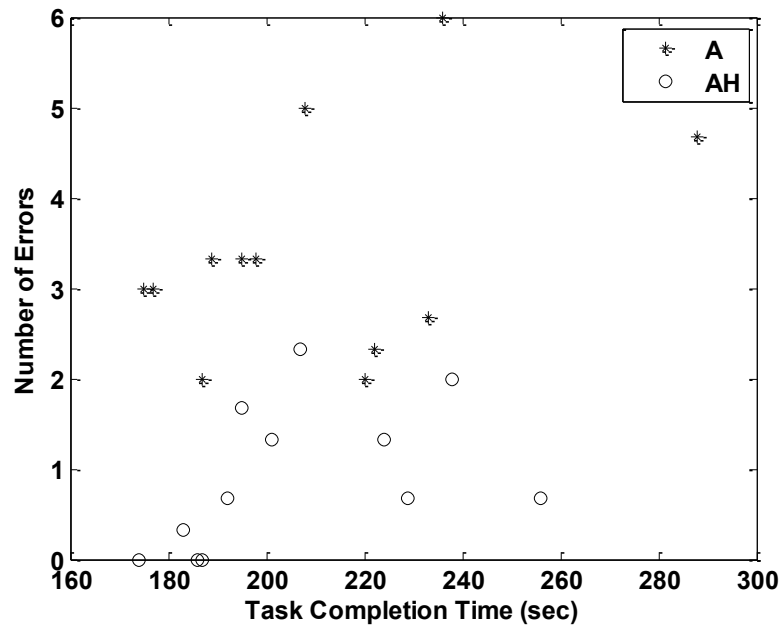

Figure 4. The task completion time versus the number of navigation errors. 


\subsection{Subjective Evaluation}

At the end of each session, the subjects were given a questionnaire related to their experience of the system. In addition to some demographic questions, there were 12 questions in the questionnaire. Some of the questions were rephrased, and asked again within the questionnaire in random order. For the answers, a 5-point Likert scale was used (1: Strongly disagree, 5: Strongly agree). For the evaluation, the average response of the subjects to the questions was utilized. The questions can be categorized into 4 groups:

Performance: 1 question was about the self-evaluation. Subjects were asked if they performed well in the experiment.

Comfort and Ease of Use: 5 questions investigated how comfortable and easy to use the driving simulator was.

Navigation Cues: 3 questions investigated whether the subjects perceived and correctly interpreted the auditory and tactile cues.

Noise and Distraction: 3 questions investigated whether the noise (i.e. city noise and music displayed from the speakers) and the distraction given by the experimenter (i.e. questioning during driving) affected the subjects' performance and attention.

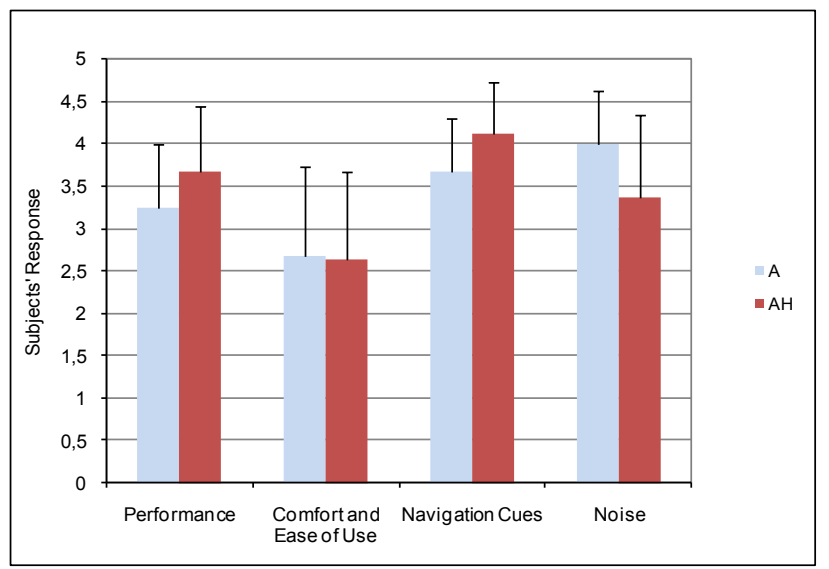

Figure 5. The results of the questionnaire for auditory and auditoryhaptic conditions. The bars represent the standard deviations.

The results are shown in Figure 5. The results show that there was no significant difference between the auditory and the auditory-haptic conditions in terms of the performance and the comfort and ease of use. However, the subjects reported that they perceived and interpreted the navigation cues better in auditoryhaptic condition than the auditory condition alone $(\mathrm{p}<0.005)$. Also, the noise and the distraction appear to have more adverse effect on their navigation performance and attention in auditory condition than in auditory-haptic condition $(\mathrm{p}<0.005)$.

\section{Conclusion}

We mounted 2 small vibration motors onto the steering wheel of a driving simulator and tested the effect of displaying vibrotactile cues in tandem with auditory ones on the navigation performance of the user. It is important to emphasize that our aim was not to improve the driving accuracy of the users as in some of the earlier studies cited in [2], but rather to enhance their recognition and interpretation of the navigation cues displayed through a GPS- based system. In particular, our hypothesis was to show the benefits of such a system when there was external noise and distraction in the environment. The results of our experiments with 12 subjects showed that, the navigation errors (making a wrong turn or taking a wrong exit) were reduced significantly when vibrotactile feedback was displayed to the users in tandem with the voice commands (Fig. 2b). Moreover, the subjective evaluation performed through a questionnaire also supported this result. The subjects perceived that their navigation performance was better in auditory-haptic condition than that of the auditory condition alone. Also, they claimed that they were affected less from noise and distractive factors when the vibrotactile cues were available for navigation.

In the future, we plan to perform some field studies to further investigate the effectiveness of the proposed approach in real-life settings. Our current design requires that the driver must hold the steering wheel with both hands from the specific locations on the wheel where the motors are mounted. However, a car driver may grip the steering wheel at random locations on the wheel or sometimes steers the car with one hand. A tactile sensor can be utilized to locate the hands of the driver on the steering wheel to display vibrotactile navigation signals accordingly. For the case of driving with one hand, tactile stimulus coded in spatio-temporal patterns can be considered as suggested in [5].

\section{REFERENCES}

[1] A. Gallace, H. Z. Tan and C. Spence. The Body Surface as a Communication System: The State of the Art after 50 Years. Presence, vol. 16, no. 6, pp. 655-676, 2007.

[2] C. Spence, C. Ho. Tactile and Multisensory Spatial Warning Signals for Drivers. IEEE Transactions on Haptics, vol. 1, no. 2, pp. 121129,2008

[3] M. Higuchi and P. Raksincharoensak. Seat Vibrotactile Warning Interface for Forward Vehicle Collision Avoidance. SICE Annual Conference, Taipei, Taiwan, 2010.

[4] J. H. Hogema, S. C. De Vries, J. B. F. Van Erp and R. J. Kiefer. A Tactile Seat for Direction Coding in Car Driving: Field Evaluation. IEEE Transactions on Haptics, vol. 2, no. 4, pp. 181-188, 2009.

[5] S. Hwang and J. Ryu. The Haptic Steering Wheel: Vibro-tactile based Navigation for the Driving Environment. IEEE International Conference on PERCOM Workshops, pp. 660-665, 2010.

[6] J. B. F. Van Erp and H. A. H. C. Van Veen. Vibrotactile In-Vehicle Navigation System. Transportation Research Part F: Traffic Psychology and Behavior, vol. 7, no. 4-5, pp. 247-256, 2004.

[7] W. B. Verwey, K. A. Brookhuis and W. H. Jansen. Safety aspects of in-vehicle information systems. Report TM-96-c002, TNO Human Factors Research Institute, Soesterberg, The Netherlands, 1996.

[8] W. H. Jansen, N.Kaptein and M. Claessens. Behavior and safety when driving with in-vehicle devices that provide real-time traffic information. In Proceedings of ITS 99, Toronto, Canada, 2000.

[9] J. B. F. Van Erp. Guidelines for the Use of Vibro-Tactile Displays in Human Computer Interaction. Proceedings of Eurohaptics, pp. 1822, 2002.

[10] J. B. F. Van Erp and H. A. H. C. Van Veen. Vibro-Tactile Information Presentation in Automobiles. Proceedings of Eurohaptics, pp. 99-104, 2001. 